\title{
Two sites of action for synapsin domain $E$ in regulating neurotransmitter release
}

Sabine Hilfiker, Felix E. Schweizer, Hung-Teh Kao, Andrew J. Czernik, Paul Greengard and George J. Augustine Nature Neurosci. 1, 29-35 (1998).

In preparing Fig. 2a, the same presynaptic traces were inadvertently used for all three panels. The correct version, which is virtually identical to our published Fig. $2 \mathrm{a}$, is shown here. The authors regret the error.
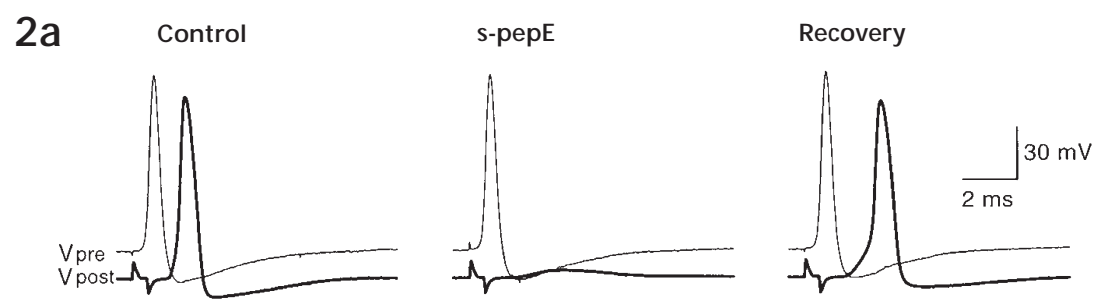

Also, because of an editorial error, the $\mathrm{x}$-axes in Figs $2 \mathrm{~b}, 2 \mathrm{c}, 2 \mathrm{~d}, 3 \mathrm{a}, 3 \mathrm{~b}, 3 \mathrm{e}$ and $5 \mathrm{c}$ were mislabeled as 'ms.' The labels should read 'min.' 


\section{Visual search for motion-in-depth: stereomotion does not 'pop out' from disparity noise}

Julie M. Harris ${ }^{1}$, Suzanne P. McKee ${ }^{2}$ and Scott N. J. Watamaniuk ${ }^{3}$

Nature Neurosci. 1, 165-168 (1998).

Because of an editorial error, the affiliation given for JMH was incomplete. The correct affiliations are:

${ }^{1}$ Address until 30 June 1998: Department of Pharmacology and Centre for Neuroscience, University of Edinburgh, Crichton St., Edinburgh, EH8 9LE, UK
Address after 1 July 1998: Department of Psychology, Ridley Building, Claremont Place, University of Newcastle, Newcastle upon Tyne, NE1 7RU, UK

\section{Visual features that vary together over time group together over space}

David Alais, Randolph Blake and Sang-Hun Lee

Nature Neurosci. 1, 160-164 (1998).

The following paragraphs were inadvertently omitted from the results section. The end of that section should read:

(page 162)...More importantly, correlated contrast modulation still produced greater coherence compared to the uncorrelated case even when the two gratings differed in contrast.

Contrast modulation of a drifting grating introduces additional temporal frequencies in the stimulus, besides those associated with the smooth drift rate. Because the contrast modulations were purposefully random, the fluctuations in temporal frequency were also random. In the case of our two-component plaid, random contrast modulations effectively modulate the length (but not the direction) of the component vectors, and these random fluctuations in vector length could be perceived as irregularities in speed, or motion 'jerkiness.' For uncorrelated contrast modulations, the changes in vector length would differ for the two components and, therefore, possibly complicate the grouping process. But why would irregular, jerky motion that is correlated among components increase perceived coherence, the result we obtained? One might argue that correlated jerkiness leads the visual system intelligently to deduce that the jerky components belong to the same object.

To test this possibility, we created a plaid consisting of two components that did not modulate in contrast. In two conditions, we explicitly selected a new drift-rate every $40 \mathrm{~ms}$ from a set of seven ranging from $4-8 \mathrm{~Hz}$; this caused motion to appear jerky. In one of these conditions, the random fluctuations in drift-rate were correlated, and in the other they were uncorrelated. In a third condition, the component gratings drifted smoothly at a constant rate of $6 \mathrm{~Hz}$ throughout the observation period. Observers again tracked periods of global coherence during 60-second observation periods. Jerky motion had no effect on perceived coherence, which was equivalent for all three conditions. Accordingly, we conclude that jerkiness is not responsible for the increased coherence produced by correlated contrast modulations.

\section{Cocaine self-administration in dopamine-transporter knockout mice}

Beatriz A. Rocha, Fabio Fumagalli, Raul R. Gainetdinov, Sara R. Jones, Robert Ator, Bruno Giros, Gary W. Miller and Marc G. Caron

Nature Neurosci. 1, 132-137 (1998).

On page 134, Fig. 4 was inadvertently printed in black and white instead of color. The corrected version is printed below.
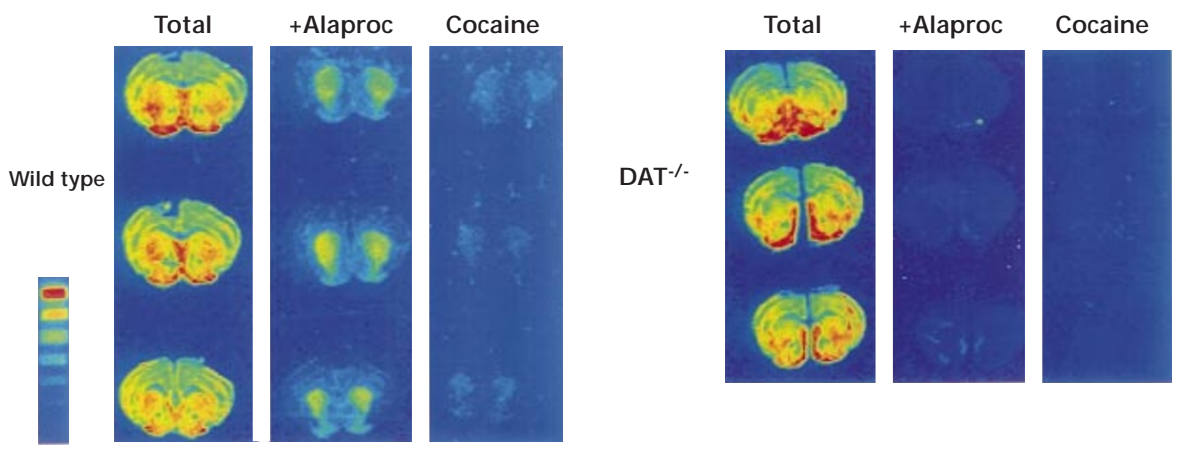


\section{Presynaptic modulation of CA3 network activity}

Kevin J. Staley, Mark Longacher, Jaideep S. Bains and Audrey Yee

Nature Neurosci. 1, 201-209 (1998).

Because of a printing error, Figs $4 c, 5 c$ and 6 were not properly reproduced. The corrected versions are printed below.

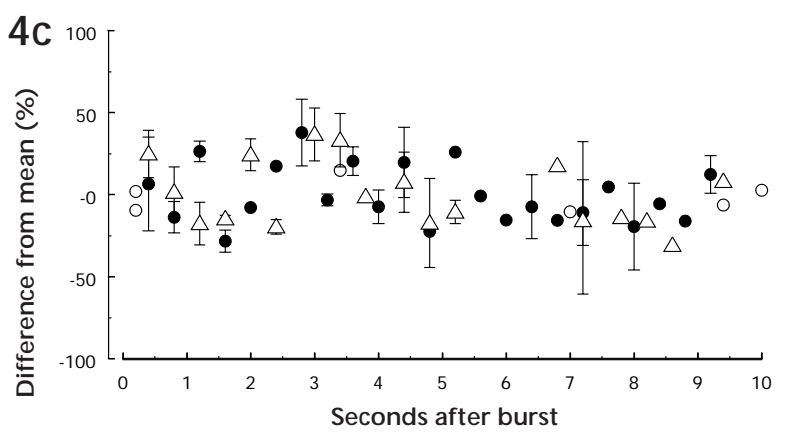

6a

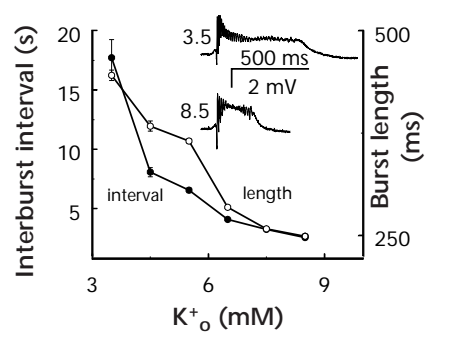

C
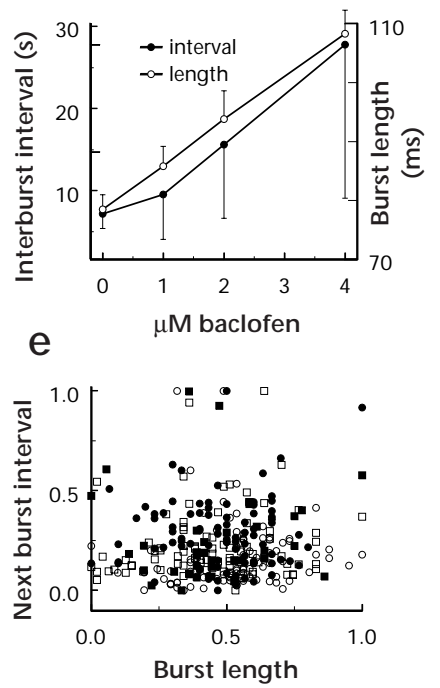

b

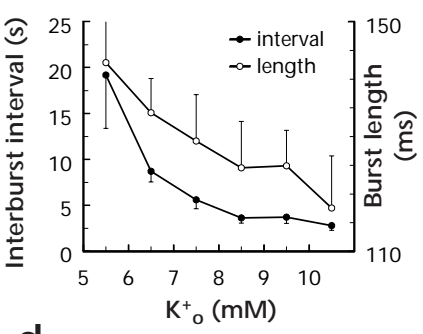

d
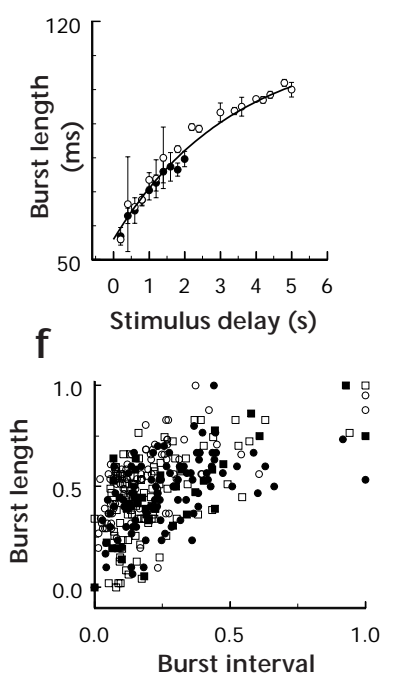

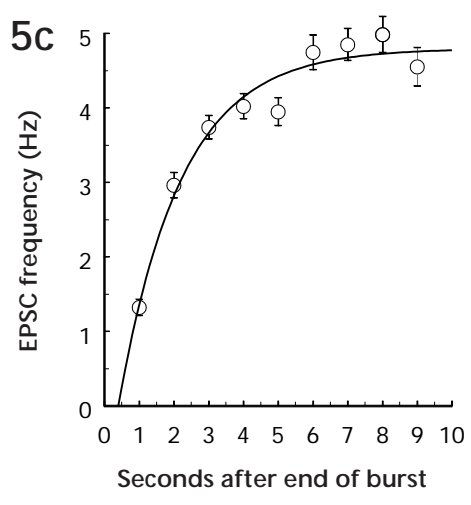

In addition, the penultimate sentence in the legend to Fig. $4 \mathrm{~b}$ was inadvertently omitted. The corrected legend should read:

Fig. 4..... (b) The experiment shown in (a) was repeated in a slice in which CA3 bursting was induced by tetanic stimulation of the CA3 pyramidal cell layer. The average interburst interval in this preparation was 13 seconds, which permitted longer delays between the end of a burst and glutamate application. As in (a), glutamate application at delays closer to the interburst interval triggered a burst more rapidly, but the initial response to glutamate was unchanged (inset). In this cell, bursts triggered calcium escape spikes. Glutamate was applied after every fourth burst in (b) and (c). 